CLNS 98/1574

CLEO 98-11

\title{
First Observation of the Decay $\tau^{-} \rightarrow K^{*-} \eta \nu_{\tau}$
}

\author{
CLEO Collaboration
}

(January 13, 2018)

\begin{abstract}
The decay $\tau^{-} \rightarrow K^{*-} \eta \nu_{\tau}$ has been observed with the CLEO II detector. The $K^{*-}$ is reconstructed in two decay channels, $K^{*-} \rightarrow K_{S} \pi^{-} \rightarrow \pi^{-} \pi^{+} \pi^{-}$and $K^{*-} \rightarrow K^{-} \pi^{0}$. The $\eta$ is reconstructed from the decay $\eta \rightarrow \gamma \gamma$. The measured branching fraction is $\mathcal{B}\left(\tau^{-} \rightarrow K^{*-} \eta \nu_{\tau}\right)=(2.9 \pm 0.8 \pm 0.4) \times 10^{-4}$. We also measure the inclusive branching fractions without requiring the $K^{*}$ resonance, $\mathcal{B}\left(\tau^{-} \rightarrow K_{S} \pi^{-} \eta \nu_{\tau}\right)=(1.10 \pm 0.35 \pm 0.11) \times 10^{-4}$ and $\mathcal{B}\left(\tau^{-} \rightarrow K^{-} \pi^{0} \eta \nu_{\tau}\right)=$ $(1.77 \pm 0.56 \pm 0.71) \times 10^{-4}$. The results indicate that the $K^{*-}$ resonance dominates the $K_{S} \pi^{-}$mass spectrum.
\end{abstract}


M. Bishai, ${ }^{1}$ S. Chen, ${ }^{1}$ J. Fast, ${ }^{1}$ J. W. Hinson, ${ }^{1}$ N. Menon, ${ }^{1}$ D. H. Miller,${ }^{1}$ E. I. Shibata,${ }^{1}$

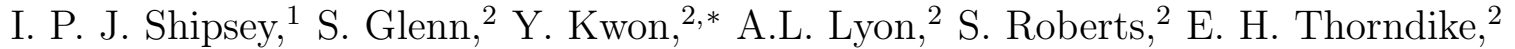
C. P. Jessop ${ }^{3}$ K. Lingel, ${ }^{3}$ H. Marsiske, ${ }^{3}$ M. L. Perl, ${ }^{3}$ V. Savinov ${ }^{3}$ D. Ugolini, ${ }^{3}$ X. Zhou, ${ }^{3}$ T. E. Coan, ${ }^{4}$ V. Fadeyev, ${ }^{4}$ I. Korolkov ${ }^{4}$ Y. Maravin, ${ }^{4}$ I. Narsky, ${ }^{4}$ R. Stroynowski, ${ }^{4}$ J. Ye, ${ }^{4}$ T. Wlodek,${ }^{4}$ M. Artuso,${ }^{5}$ E. Dambasuren,${ }^{5}$ S. Kopp,${ }^{5}$ G. C. Moneti,${ }^{5}$ R. Mountain,${ }^{5}$ S. Schuh, ${ }^{5}$ T. Skwarnicki, ${ }^{5}$ S. Stone ${ }^{5}$ A. Titov,${ }^{5}$ G. Viehhauser ${ }^{5}$ J.C. Wang, ${ }^{5}$ J. Bartelt ${ }^{6}$ S. E. Csorna, ${ }^{6}$ K. W. McLean, ${ }^{6}$ S. Marka,${ }^{6}$ Z. Xu, ${ }^{6}$ R. Godang, ${ }^{7}$ K. Kinoshita, ${ }^{7}$ I. C. Lai, ${ }^{7}$ P. Pomianowski, ${ }^{7}$ S. Schrenk,${ }^{7}$ G. Bonvicini,${ }^{8}$ D. Cinabro,${ }^{8}$ R. Greene,${ }^{8}$ L. P. Perera,${ }^{8}$ G. J. Zhou, ${ }^{8}$ S. Chan,${ }^{9}$ G. Eigen,${ }^{9}$ E. Lipeles,${ }^{9}$ J. S. Miller, ${ }^{9}$ M. Schmidtler, ${ }^{9}$ A. Shapiro, ${ }^{9}$ W. M. Sun, ${ }^{9}$ J. Urheim, ${ }^{9}$ A. J. Weinstein, ${ }^{9}$ F. Würthwein, ${ }^{9}$ D. E. Jaffe, ${ }^{10}$ G. Masek, ${ }^{10}$ H. P. Paar, ${ }^{10}$ E. M. Potter,${ }^{10}$ S. Prell, ${ }^{10}$ V. Sharma, ${ }^{10}$ D. M. Asner, ${ }^{11}$ J. Gronberg, ${ }^{11}$ T. S. Hill, ${ }^{11}$ D. J. Lange, ${ }^{11}$ R. J. Morrison, ${ }^{11}$ H. N. Nelson, ${ }^{11}$ T. K. Nelson, ${ }^{11}$ D. Roberts, ${ }^{11}$ B. H. Behrens, ${ }^{12}$ W. T. Ford, ${ }^{12}$ A. Gritsan, ${ }^{12}$ H. Krieg, ${ }^{12}$ J. Roy, ${ }^{12}$ J. G. Smith, ${ }^{12}$ J. P. Alexander ${ }^{13}$ R. Baker ${ }^{13}$ C. Bebek, ${ }^{13}$ B. E. Berger,${ }^{13}$ K. Berkelman, ${ }^{13}$ V. Boisvert,${ }^{13}$

D. G. Cassel, ${ }^{13}$ D. S. Crowcroft, ${ }^{13}$ M. Dickson, ${ }^{13}$ S. von Dombrowski, ${ }^{13}$ P. S. Drell, ${ }^{13}$ K. M. Ecklund, ${ }^{13}$ R. Ehrlich, ${ }^{13}$ A. D. Foland,${ }^{13}$ P. Gaidarev, ${ }^{13}$ R. S. Galik, ${ }^{13}$ L. Gibbons, ${ }^{13}$ B. Gittelman, ${ }^{13}$ S. W. Gray, ${ }^{13}$ D. L. Hartill, ${ }^{13}$ B. K. Heltsley, ${ }^{13}$ P. I. Hopman,,${ }^{13}$

J. Kandaswamy, ${ }^{13}$ D. L. Kreinick, ${ }^{13}$ T. Lee, ${ }^{13}$ Y. Liu, ${ }^{13}$ N. B. Mistry, ${ }^{13}$ C. R. Ng, ${ }^{13}$ E. Nordberg, ${ }^{13}$ M. Ogg, ${ }^{13}$, J. R. Patterson, ${ }^{13}$ D. Peterson, ${ }^{13}$ D. Riley, ${ }^{13}$ A. Soffer, ${ }^{13}$ B. Valant-Spaight, ${ }^{13}$ A. Warburton, ${ }^{13}$ C. Ward,${ }^{13}$ M. Athanas,${ }^{14}$ P. Avery, ${ }^{14}$ C. D. Jones, ${ }^{14}$ M. Lohner, ${ }^{14}$ C. Prescott, ${ }^{14}$ A. I. Rubiera, ${ }^{14}$ J. Yelton, ${ }^{14}$ J. Zheng, ${ }^{14}$ G. Brandenburg, ${ }^{15}$ R. A. Briere, ${ }^{15}$ A. Ershov, ${ }^{15}$ Y. S. Gao, ${ }^{15}$ D. Y.-J. Kim,${ }^{15}$ R. Wilson, ${ }^{15}$ H. Yamamoto, ${ }^{15}$ T. E. Browder ${ }^{16}$ Y. Li, ${ }^{16}$ J. L. Rodriguez ${ }^{16}$ S. K. Sahu, ${ }^{16}$ T. Bergfeld, ${ }^{17}$ B. I. Eisenstein, ${ }^{17}$ J. Ernst, ${ }^{17}$ G. E. Gladding, ${ }^{17}$ G. D. Gollin, ${ }^{17}$ R. M. Hans, ${ }^{17}$ E. Johnson, ${ }^{17}$ I. Karliner, ${ }^{17}$ M. A. Marsh ${ }^{17}$ M. Palmer, ${ }^{17}$ M. Selen, ${ }^{17}$ J. J. Thaler,${ }^{17}$ K. W. Edwards, ${ }^{18}$ A. Bellerive, ${ }^{19}$

R. Janicek, ${ }^{19}$ P. M. Patel, ${ }^{19}$ A. J. Sadoff, ${ }^{20}$ R. Ammar, ${ }^{21}$ P. Baringer, ${ }^{21}$ A. Bean, ${ }^{21}$

D. Besson ${ }^{21}$ D. Coppage ${ }^{21}$ C. Darling, ${ }^{21}$ R. Davis,${ }^{21}$ S. Kotov ${ }^{21}$ I. Kravchenko, ${ }^{21}$ N. Kwak,${ }^{21}$ L. Zhou, ${ }^{21}$ S. Anderson, ${ }^{22}$ Y. Kubota, ${ }^{22}$ S. J. Lee, ${ }^{22}$ R. Mahapatra, ${ }^{22}$ J. J. O'Neill, ${ }^{22}$ R. Poling, ${ }^{22}$ T. Riehle, ${ }^{22}$ A. Smith, ${ }^{22}$ M. S. Alam, ${ }^{23}$ S. B. Athar, ${ }^{23}$ Z. Ling, ${ }^{23}$ A. H. Mahmood, ${ }^{23}$ S. Timm,${ }^{23}$ F. Wappler, ${ }^{23}$ A. Anastassov, ${ }^{24}$ J. E. Duboscq, ${ }^{24}$ K. K. Gan ${ }^{24}$ T. Hart,${ }^{24}$ K. Honscheid,${ }^{24}$ H. Kagan,${ }^{24}$ R. Kass,${ }^{24}$ J. Lee,${ }^{24}$ H. Schwarthoff,${ }^{24}$ A. Wolf, ${ }^{24}$ M. M. Zoeller, ${ }^{24}$ S. J. Richichi, ${ }^{25}$ H. Severini, ${ }^{25}$ P. Skubic ${ }^{25}$ and A. Undrus ${ }^{25}$

\footnotetext{
${ }^{1}$ Purdue University, West Lafayette, Indiana 47907

${ }^{2}$ University of Rochester, Rochester, New York 14627

${ }^{3}$ Stanford Linear Accelerator Center, Stanford University, Stanford, California 94309

${ }^{4}$ Southern Methodist University, Dallas, Texas 75275

${ }^{5}$ Syracuse University, Syracuse, New York 13244

${ }^{6}$ Vanderbilt University, Nashville, Tennessee 37235

${ }^{7}$ Virginia Polytechnic Institute and State University, Blacksburg, Virginia 24061
}

\footnotetext{
*Permanent address: Yonsei University, Seoul 120-749, Korea.

${ }^{\dagger}$ Permanent address: University of Texas, Austin TX 78712.
} 
${ }^{8}$ Wayne State University, Detroit, Michigan 48202

${ }^{9}$ California Institute of Technology, Pasadena, California 91125

${ }^{10}$ University of California, San Diego, La Jolla, California 92093

${ }^{11}$ University of California, Santa Barbara, California 93106

${ }^{12}$ University of Colorado, Boulder, Colorado 80309-0390

${ }^{13}$ Cornell University, Ithaca, New York 14853

${ }^{14}$ University of Florida, Gainesville, Florida 32611

${ }^{15}$ Harvard University, Cambridge, Massachusetts 02138

${ }^{16}$ University of Hawaii at Manoa, Honolulu, Hawaii 96822

${ }^{17}$ University of Illinois, Urbana-Champaign, Illinois 61801

${ }^{18}$ Carleton University, Ottawa, Ontario, Canada K1S 5B6 and the Institute of Particle Physics, Canada

${ }^{19}$ McGill University, Montréal, Québec, Canada H3A 2T8 and the Institute of Particle Physics, Canada

${ }^{20}$ Ithaca College, Ithaca, New York 14850

${ }^{21}$ University of Kansas, Lawrence, Kansas 66045

${ }^{22}$ University of Minnesota, Minneapolis, Minnesota 55455

${ }^{23}$ State University of New York at Albany, Albany, New York 12222

${ }^{24}$ Ohio State University, Columbus, Ohio 43210

${ }^{25}$ University of Oklahoma, Norman, Oklahoma 73019 
The study of the hadronic decays of the $\tau$ lepton is important for a better understanding of the weak hadronic current and its symmetries. The decays involving an $\eta$ meson are associated with the Wess-Zumino-Witten anomaly [1] and are rare. The first such decay, $\tau^{-} \rightarrow \pi^{-} \pi^{0} \eta \nu_{\tau}$, was observed by CLEO in 1992 [2] and subsequently by ALEPH [3]. More recently, CLEO has measured the branching fractions of two other decays [4], $\mathcal{B}\left(\tau^{-} \rightarrow\right.$ $\left.K^{-} \eta \nu_{\tau}\right)=(2.6 \pm 0.5 \pm 0.5) \times 10^{-4}$ [5] and $\mathcal{B}\left(\tau^{-} \rightarrow(3 h)^{-} \eta \nu_{\tau}\right)=\left(3.5_{-0.6}^{+0.7} \pm 0.7\right) \times 10^{-4}$ [6], where $h=\pi$ or $K$. Both measurements are two orders of magnitude higher than the predictions by Pich [7] based on chiral perturbation theory. However, the recent calculation by Li [8] using an effective chiral theory in the limit of chiral symmetry is in good agreement with these results. In the calculation, the former decay proceeds through the vector current with $K^{*}$ dominant and the latter decay proceeds through the axial-vector current with $a_{1}$ dominant. For the decay $\tau^{-} \rightarrow(K \pi)^{-} \eta \nu_{\tau}$, Pich predicts that the $K^{*}$ enhancement in the $K \pi$ system with $\mathcal{B}\left(\tau^{-} \rightarrow K^{-} \pi^{0} \eta \nu_{\tau}\right) \sim 8.8 \times 10^{-6}$ and $\mathcal{B}\left(\tau^{-} \rightarrow \pi^{-} \bar{K}^{0} \eta \nu_{\tau}\right) \sim 2.2 \times 10^{-5}$ while Li predicts that the $K_{1}$ axial-vector current is dominant with $\mathcal{B}\left(\tau^{-} \rightarrow K^{*-} \eta \nu_{\tau}\right)=1.01 \times 10^{-4}$. In this Letter, we report a first measurement of the decay $\tau^{-} \rightarrow K^{*-} \eta \nu_{\tau}, K^{*-} \rightarrow K_{S} \pi^{-}$and $K^{*-} \rightarrow K^{-} \pi^{0}$. We also measure the inclusive branching fractions without requiring the $K^{*}$ resonance.

The data used in this analysis have been collected from $e^{+} e^{-}$collisions at a center-ofmass energy of $E_{c m}=10.6 \mathrm{GeV}$ with the CLEO II detector at the Cornell Electron Storage Ring (CESR). The total integrated luminosity of the sample is $4.7 \mathrm{fb}^{-1}$, corresponding to the production of $4.3 \times 10^{6} \tau$ pairs. The CLEO II detector has been described in detail elsewhere [9].

We select $\tau^{+} \tau^{-}$events in which one charged particle from the tag $\tau$ decay is recoiling against one or three charged particles of the signal decay. The candidate events must therefore have two or four charged tracks and zero net charge. To reject beam-gas events, we require that the distance of closest approach to the $e^{+} e^{-}$interaction point of the non- $K_{S}$ candidate tracks be within $0.5 \mathrm{~cm}(5 \mathrm{~cm})$ transverse to (along) the beam direction. Each event is divided into two hemispheres (tag vs. signal) using the plane perpendicular to the thrust axis [10], calculated from both charged tracks and photons. Photons are defined as energy clusters in the calorimeter of at least $60 \mathrm{MeV}$ in the barrel, $|\cos \theta|<0.80$, and 100 $\mathrm{MeV}$ in the end cap, $0.80<|\cos \theta|<0.95$, where $\theta$ is the polar angle with respect to the beam axis. There must be two or more photons in the barrel for the signal hemisphere. However, if there are more than two (four) photons with an energy above $100 \mathrm{MeV}$, including the end cap, the event is rejected in the $\tau^{-} \rightarrow K_{S} \pi^{-} \eta \nu_{\tau}\left(\tau^{-} \rightarrow K^{-} \pi^{0} \eta \nu_{\tau}\right)$ analysis. The opening angle between the total momentum vectors of the decay products of the two $\tau$ leptons must be greater than $120^{\circ}$. The tag hemisphere must contain only one charged particle, and its momentum must be greater than $0.5 \mathrm{GeV} / \mathrm{c}$. The hemisphere may not contain more than three energetic photons $(E>100 \mathrm{MeV})$. In the case of two or more photons, there must be at least one $\pi^{0}$ candidate reconstructed, $\left|M_{\gamma \gamma}-M_{\pi^{0}}\right|<20 \mathrm{MeV} / \mathrm{c}^{2}(\sim 3 \sigma)$. The hadronic background is suppressed by a requirement that the total invariant mass of the particles in each hemisphere be less than the $\tau$ mass, $M<1.78 \mathrm{GeV} / \mathrm{c}^{2}$. Two-photon, Bhabha, and hadronic events are suppressed by the requirements on the total visible energy, $0.25<E_{t o t} / E_{c m}<0.85$, and on the measured net transverse momentum of the event, $p_{\perp}>0.3 \mathrm{GeV} / \mathrm{c}$. All charged particles and photons are included in the calculation of these kinematic variables. 
Particle identification for the $\tau^{-} \rightarrow K^{-} \pi^{0} \eta \nu_{\tau}$ decay is based on a confidence level ratio which is constructed from the confidence levels for $\pi$ and $K$ hypotheses [5], $C L_{\pi}$ and $C L_{K}$. The confidence level ratio for $K$ is $R_{K}=C L_{K} /\left(C L_{\pi}+C L_{K}\right)$, and similarly for $\pi\left(R_{\pi}=\right.$ $\left.1-R_{K}\right)$. The confidence level is computed from the $\chi^{2}$ probability for a particle hypothesis using a combination of the time of flight and drift chamber $(d E / d x)$ information.

Candidate $K_{S}$ mesons are reconstructed using pairs of oppositely charged tracks with vertices separated from the primary interaction point by at least $10 \mathrm{~mm}$ in the plane transverse to the beam. The $\pi^{+} \pi^{-}$invariant mass is required to be within $15 \mathrm{MeV} / \mathrm{c}^{2}(\sim 3 \sigma)$ of the $K_{S}$ mass.

The $\eta$ mesons are reconstructed with photons in the barrel using the $\gamma \gamma$ decay channel. Each photon must have an energy above $150 \mathrm{MeV}$ and a lateral profile of energy deposition consistent with that expected of a photon. In addition, we do not use the fragments of a nearby large shower. The photon may not combine with any other photon to form a $\pi^{0}$ candidate.

For the $\tau^{-} \rightarrow K^{*-} \eta \nu_{\tau} \rightarrow K_{S} \pi^{-} \eta \nu_{\tau}$ analysis, events with three charged particles in the signal hemisphere were selected. Figure 1 shows the invariant mass spectra of two photons accompanying the $K_{S}$ candidate, with the requirements that the $K_{S} \pi^{-}$mass be in the $K^{*-}$ signal band $\left(0.81-0.97 \mathrm{GeV} / \mathrm{c}^{2}\right)$ or sidebands $\left(0.70-0.78,1.00-1.08 \mathrm{GeV} / \mathrm{c}^{2}\right)$. An $\eta$ signal is observed in the $K^{*-}$ signal region, and there is no indication of a signal in the sideband region. The curves show fits to the data using a Gaussian signal and a linear background. The width of the Gaussian is constrained to the Monte Carlo expectation, $\sigma=14 \mathrm{MeV} / \mathrm{c}^{2}$. The fit shown in Fig. 1(a) yields a signal of $13.3 \pm 3.9$ events. The $\eta$ yield in the $K^{*-}$ sidebands is $1.0_{-1.0}^{+1.7}$ events. We have therefore observed for the first time the decay $\tau^{-} \rightarrow K^{*-} \eta \nu_{\tau}$.

As a check of the validity of the signal for $\tau^{-} \rightarrow K^{*-} \eta \nu_{\tau}$, we show the invariant mass spectrum of the $K_{S} \pi^{-}$system for events with an $\eta$ candidate $\left(\left|M_{\gamma \gamma}-M_{\eta}\right|<45 \mathrm{MeV} / \mathrm{c}^{2}\right)$ in Fig. 2. A clear $K^{*-}$ signal is observed.

For the $\tau^{-} \rightarrow K^{*-} \eta \nu_{\tau} \rightarrow K^{-} \pi^{0} \eta \nu_{\tau}$ analysis, we select events with the signal hemisphere containing a charged particle, a $\pi^{0}$ candidate reconstructed using barrel photons plus two other barrel photons. The $R_{K}$ distributions for the charged particle in the signal hemisphere is shown in Fig. 3. The invariant mass of the two photons accompanying the charge particle and $\pi^{0}$ candidate is required to be (a) in the $\eta$ signal band $\left(0.50-0.59 \mathrm{GeV} / \mathrm{c}^{2}\right)$, and (b) in the $\eta$ signal sideband $\left(0.440-0.485,0.605-0.650 \mathrm{GeV} / \mathrm{c}^{2}\right)$. Figures $3(\mathrm{c})$ and $3(\mathrm{~d})$ show the corresponding distributions for the case in which the $K^{-} \pi^{0}$ mass is in the $K^{*-}$ signal band with the assumption that the charged particle is a kaon. There are enhancements at $R_{K}=$ 0 and, in (a) and (c), 1.0, as expected from the decays $\tau^{-} \rightarrow \pi^{-} \pi^{0} \eta \nu_{\tau}$ and $\tau^{-} \rightarrow K^{-} \pi^{0} \eta \nu_{\tau}$ respectively. The histograms show fits to the data using the Monte Carlo expectation for $R_{K}$ spectra for these two decays and the migration from other $\tau$ decays. The fit results on the number of events with a kaon accompanying the $\eta$ candidate are summarized in Table $\mathbb{\square}$.

The detection efficiencies for the candidate events and background from hadronic events are calculated with a Monte Carlo simulation. The KORALB program [11] is used to generate $\tau^{+} \tau^{-}$pairs and the Lund program [12] for hadronic events. The signal decays are modeled by phase space assuming a $V-A$ weak interaction. The detector response is simulated using the GEANT program [13]. The identification and misidentification efficiencies of pions and kaons are calibrated as a function of momentum by comparing the efficiencies measured from samples of pions and kaons from the decays $D^{*+} \rightarrow D^{0} \pi^{+} \rightarrow K^{-} \pi^{+} \pi^{+}$and $K_{S} \rightarrow \pi^{+} \pi^{-}$ 


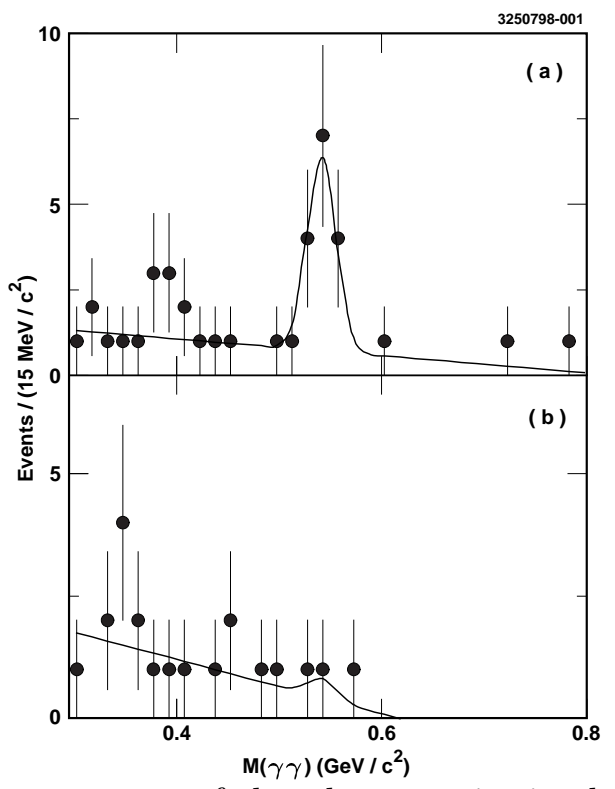

FIG. 1. The invariant mass spectrum of the photon pairs in the signal hemisphere containing a $K_{S}$ candidate. The $K_{S} \pi^{-}$invariant mass is required to be in the $K^{*-}$ signal band in (a) and in the $K^{*-}$ sideband in (b). The curves show fits to the data.

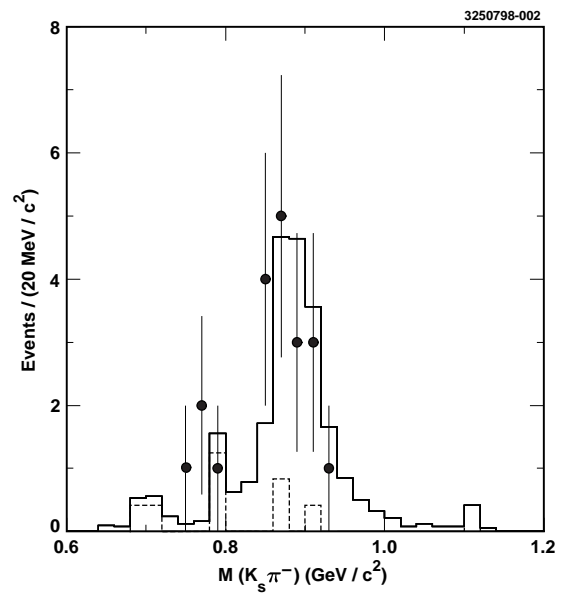

FIG. 2. The invariant mass spectrum of the $K_{S} \pi^{-}$system in the signal hemisphere containing an $\eta$ candidate. The histograms show the Monte Carlo expectation, including the hadronic background (dotted). 
with the hadronic Monte Carlo expectations. In the estimation of the hadronic background, the $\eta$ multiplicity in the hadronic Monte Carlo program has been normalized to produce the observed multiplicity in events with the invariant mass of one of the hemispheres greater than $M_{\tau}$. Two-photon interactions are estimated to be a negligible source of background [5].

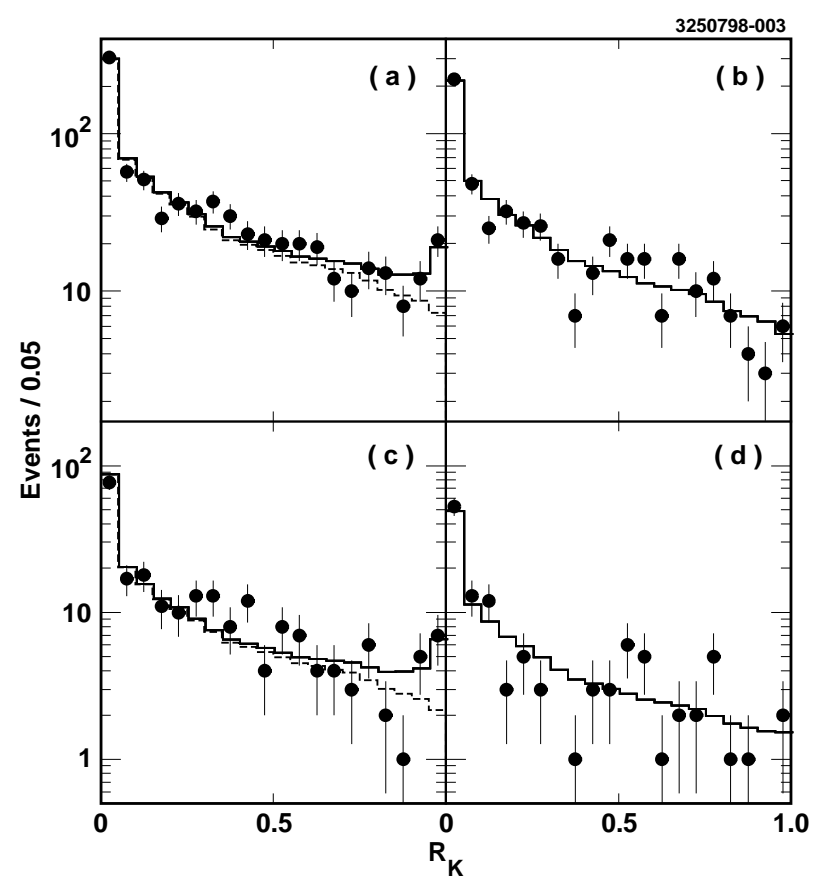

FIG. 3. The $R_{K}$ spectrum of the charged particle in the signal hemisphere containing the $\eta$ candidate. The invariant mass of the two photons accompanying the charged particle and $\pi^{0}$ candidate is required to be in the $\eta$ signal band in (a) and in the $\eta$ sideband in (b). Assuming the charged particle is a kaon, the $K^{-} \pi^{0}$ mass is required to be in the $K^{*}$ band in (c) and (d). The histograms show fits to the data with the pions contribution indicated by the dashed histograms.

TABLE I. Number of events with a charged kaon from the fits of Fig. 3 together with the $\chi^{2}$ per degree of freedom.

\begin{tabular}{lccc}
\hline \hline Requirements & Fig. 䀚 & $N_{K}$ & $\chi^{2} / \mathrm{DOF}$ \\
\hline$\eta$ signal band & (a) & $36.4 \pm 11.4$ & $23 / 18$ \\
$\eta$ sideband & (b) & $0.0_{-0.0}^{+6.7}$ & $35 / 18$ \\
$\eta$ signal band, $K^{*}$ region & (c) & $11.7 \pm 5.6$ & $25 / 18$ \\
$\eta$ sideband, $K^{*}$ region & (d) & $1.0_{-1.0}^{+3.1}$ & $21 / 15$ \\
\hline \hline
\end{tabular}

The signals, backgrounds, and detection efficiencies are summarized in Tables [1 and [III. In calculating the detection efficiencies and backgrounds in the $K^{*-} \rightarrow K^{-} \pi^{0}$ analysis, the Monte Carlo predictions have been corrected for the appropriate momentum-dependent identification and misidentification efficiency scaling factors. The branching fraction for $\tau^{-} \rightarrow(3 h)^{-} \eta \nu_{\tau}$ [6] is used to estimate the feeddown in the $\tau^{-} \rightarrow K_{S} \pi^{-} \eta \nu_{\tau}$ analysis.

There are several sources of systematic errors as shown in Table एV. These include the uncertainties in the number of $\tau^{+} \tau^{-}$events produced, branching fractions, background subtraction, fitting procedure, $K_{S}$ detection efficiency, acceptance calculation, decay modeling, 
TABLE II. Summary of signals, backgrounds, detection efficiencies, and branching fractions for the decay $\tau^{-} \rightarrow K_{S} \pi^{-} \eta \nu_{\tau}$. All errors are statistical.

\begin{tabular}{lcc}
\hline \hline$K^{*}$ requirement & Yes & No \\
\hline Signal & $13.3 \pm 3.9$ & $15.1 \pm 4.5$ \\
Signal $\left(K^{*}\right.$ sideband $)$ & $1.0_{-1.0}^{+1.7}$ & - \\
$q \bar{q}$ & $0.0_{-0.0}^{+1.4}$ & $0.5_{-0.5}^{+0.7}$ \\
$3 h \eta$ & $0.4 \pm 0.2$ & $0.6 \pm 0.3$ \\
$3 h \eta\left(K^{*}\right.$ sideband $)$ & $0.2 \pm 0.2$ & - \\
Eff. $(\%)$ & $4.4 \pm 0.1$ & $5.5 \pm 0.1$ \\
\hline$B\left(10^{-4}\right)$ & $1.18 \pm 0.38$ & $1.10 \pm 0.35$ \\
\hline \hline
\end{tabular}

TABLE III. Summary of signals, backgrounds, detection efficiencies, and branching fractions for the decay $\tau^{-} \rightarrow K^{-} \pi^{0} \eta \nu_{\tau}$. All errors are statistical.

\begin{tabular}{lcc}
\hline \hline$K^{*}$ requirement & Yes & No \\
\hline$\eta$ band & $11.7 \pm 5.6$ & $36.4 \pm 11.4$ \\
$\eta$ sideband & $1.0_{-1.0}^{+3.1}$ & $0.0_{-0.0}^{+6.7}$ \\
$q \bar{q}$ & $<3.5 @ 90 \% C L$ & $0_{-0}^{+2}$ \\
$K^{-} \eta$ & - & $0.4 \pm 0.1$ \\
Eff. $(\%)$ & $4.6 \pm 0.1$ & $6.0 \pm 0.1$ \\
\hline$B\left(10^{-4}\right)$ & $0.69 \pm 0.36$ & $1.77 \pm 0.56$ \\
\hline \hline
\end{tabular}

TABLE IV. Summary of systematic errors (\%).

\begin{tabular}{lcc}
\hline \hline & $K_{S} \pi^{-} \eta \nu_{\tau}$ & $K^{-} \pi^{0} \eta \nu_{\tau}$ \\
\hline$N_{\tau \tau}$ & 1.4 & 1.4 \\
$\mathcal{B}(\eta \rightarrow \gamma \gamma)$ & 0.8 & 0.8 \\
$\mathcal{B}\left(\tau^{-} \rightarrow(3 h)^{-} \eta \nu_{\tau}\right)$ & 2 & - \\
Hadronic background & 5 & 6 \\
$\eta$ sideband subtraction & - & 29 \\
Fit & 5 & 27 \\
$K_{S}$ detection eff. [15] & 2 & - \\
Acceptance & 3 & 3 \\
Decay model & 4 & 4 \\
MC statistics & 3 & 2 \\
\hline Total & 10 & 40 \\
\hline \hline
\end{tabular}


as well as the uncertainty due to limited Monte Carlo statistics. The uncertainty in the $R_{K}$ spectrum at $R_{K}=1$ for pions (tail) and kaons (peak) is a major source of the systematic error in the $R_{K}$ fitting analysis. The $R_{K}$ kaon peak depends on the momentum distribution which is different for $\tau^{-} \rightarrow K^{*-} \eta \nu_{\tau}$ and non-resonant $\tau^{-} \rightarrow K^{-} \pi^{0} \eta \nu_{\tau}$ decays. The differences are taken as the systematic error estimate. The systematic error in the acceptance calculation includes the uncertainties in the simulation of the tracking, photon detection and veto efficiencies. The acceptance depends also on the decay model; the corresponding systematic error is estimated by comparing the detection efficiencies for the decays $\tau^{-} \rightarrow K_{S} \pi^{-} \eta \nu_{\tau}$ and $\tau^{-} \rightarrow K^{-} \pi^{0} \eta \nu_{\tau}$ with and without the $K^{*}$ resonance.

The branching fractions for $\tau^{-} \rightarrow K^{*-} \eta \nu_{\tau}, K^{*-} \rightarrow K_{S} \pi^{-}$and $K^{*-} \rightarrow K^{-} \pi^{0}$ are extracted after correcting for backgrounds and detection efficiencies. The results are

$$
\begin{aligned}
& \mathcal{B}\left(\tau^{-} \rightarrow K^{*-} \eta \nu_{\tau}\right) \times \mathcal{B}\left(K^{*-} \rightarrow K_{S} \pi^{-}\right)=(1.18 \pm 0.38 \pm 0.12) \times 10^{-4}, \\
& \mathcal{B}\left(\tau^{-} \rightarrow K^{*-} \eta \nu_{\tau}\right) \times \mathcal{B}\left(K^{*-} \rightarrow K^{-} \pi^{0}\right)=(0.69 \pm 0.36 \pm 0.28) \times 10^{-4}
\end{aligned}
$$

Combining these results with the isospin requirement $\mathcal{B}\left(K^{*-} \rightarrow K_{S} \pi^{-}\right)=$ $\mathcal{B}\left(K^{*-} \rightarrow K^{-} \pi^{0}\right)=1 / 3$ yields

$$
\mathcal{B}\left(\tau^{-} \rightarrow K^{*-} \eta \nu_{\tau}\right)=(2.90 \pm 0.80 \pm 0.42) \times 10^{-4} .
$$

The inclusive measurements without the $K^{*}$ resonance requirement are

$$
\begin{aligned}
& \mathcal{B}\left(\tau^{-} \rightarrow K_{S} \pi^{-} \eta \nu_{\tau}\right)=(1.10 \pm 0.35 \pm 0.11) \times 10^{-4}, \\
& \mathcal{B}\left(\tau^{-} \rightarrow K^{-} \pi^{0} \eta \nu_{\tau}\right)=(1.77 \pm 0.56 \pm 0.71) \times 10^{-4},
\end{aligned}
$$

where the first error is statistical and the second systematic. The inclusive results are in reasonable agreement with the measurements requiring the $K^{*}$ resonance.

In summary we have measured for the first time the branching fraction of $\tau^{-} \rightarrow K^{*-} \eta \nu_{\tau}$. The result is somewhat higher than the theoretical prediction by Li $80\left(1.01 \times 10^{-4}\right)$. We also measure the inclusive branching fractions without requiring the $K^{*}$ resonance. The measurements are significantly higher than the theoretical predictions by Pich [7]. The results for the $\tau^{-} \rightarrow K_{S} \pi^{-} \eta \nu_{\tau}$ mode indicate that the $K^{*-}$ resonance dominates the $K_{S} \pi^{-}$ mass spectrum.

We gratefully acknowledge the effort of the CESR staff in providing us with excellent luminosity and running conditions. This work was supported by the National Science Foundation, the U.S. Department of Energy, Research Corporation, the Natural Sciences and Engineering Research Council of Canada, the A.P. Sloan Foundation, the Swiss National Science Foundation, and the Alexander von Humboldt Stiftung. 


\section{REFERENCES}

[1] J. Wess and B. Zumino, Phys. Lett. B37, 95 (1971); E. Witten, Nucl. Phys. B223, 422 (1983).

[2] CLEO Collaboration, M. Artuso et al., Phys. Rev. Lett. 69, 3278 (1992).

[3] ALEPH Collaboration, D. Buskulic et al., Z. Phys. C74, 263 (1997).

[4] Charge conjugation is implied throughout the paper.

[5] CLEO Collaboration, J. Bartelt et al., Phys. Rev. Lett. 76, 4119 (1996).

[6] CLEO Collaboration, T.Bergfeld et al., Phys. Rev. Lett. 79, 2406 (1997).

[7] A. Pich, Phys. Lett. B196, 561 (1987).

[8] B. A. Li, Phys. Rev. D55, 1436 (1997); Phys. Rev. D57, 1790 (1998).

[9] CLEO Collaboration, Y. Kubota et al., Nucl. Instrum. Methods A320, 66 (1992).

[10] E. Farhi, Phys. Rev. Lett. 39, 1587 (1977).

[11] S. Jadach and Z. Was, Comput. Phys. Commun. 36, 191 (1985); 64, 267 (1991); S. Jadach, J.H. Kuhn, and Z. Was, ibid. 64, 275 (1991).

[12] T. Sjöstrand and M. Bengtsson, Comput. Phys. Commun. 43, 367 (1987).

[13] R. Brun et al., CERN Report No. CERN-DD/EE/84-1, 1987 (unpublished).

[14] Review of Particle Properties, R.M. Barnett et al., Phys. Rev. D54 (1996).

[15] CLEO Collaboration, M. Bishai et al., Phys. Rev. Lett. 78, 3261 (1997). 